\title{
Hidden in plain sight: Low literacy adults in a developed country overcoming social and educational challenges through mobile learning support tools
}

\author{
Cosmin Munteanu ${ }^{1}$, Heather Molyneaux ${ }^{1}$, Julie Maitland ${ }^{1}$, Daniel McDonald ${ }^{1}$, \\ Rock Leung ${ }^{2}$, Hélène Fournier ${ }^{1}$, Joanna Lumsden ${ }^{3}$ \\ ${ }^{1)}$ National Research Council \\ ${ }^{2)}$ University of British Columbia \\ ${ }^{3)}$ Aston University \\ Fredericton, Canada \\ Vancouver, Canada \\ Birmingham, UK
}

\{Cosmin.Munteanu, Heather.Molyneaux, Julie.Maitland, Daniel.McDonald\}@nrc-cnrc.gc.ca rockl@cs.ubc.ca, Helene.Fournier@nrc-cnrc.gc.ca, j.lumsden@aston.ac.uk

Corresponding author: Cosmin Munteanu

Email address: Cosmin.Munteanu@nrc-cnrc.gc.ca

Phone: 506-444-0527

Address: National Research Council of Canada

46 Dineen Dr.

Fredericton, NB, E3B 9W4

Canada

\section{Abstract}

Illiteracy is often associated with people in developing countries. However, an estimated $50 \%$ of adults in a developed country such as Canada lack the literacy skills required to cope with the challenges of today's society; for them, tasks such as reading, understanding, basic arithmetic, and using everyday items are a challenge. Many community-based organizations offer resources and support for these adults, yet overall functional literacy rates are not improving. This is due to a wide range of factors, such as poor retention of adult learners in literacy programs, obstacles in transferring the acquired skills from the classroom to the real life, personal attitudes towards learning, and the stigma of functional illiteracy.

In our research we examined the opportunities afforded by personal mobile devices in providing learning and functional support to low-literacy adults. We present the findings of an exploratory study aimed at investigating the reception and adoption of a technological solution for adult learners. ALEX $\odot$ is a mobile application designed for use both in the classroom and in daily life in order to help low-literacy adults become increasingly literate and independent. Such a solution complements literacy programs by increasing users' motivation and interest in learning, and raising their confidence levels both in their education pursuits and in facing the challenges of their daily lives. We also reflect on the challenges we faced in designing and conducting our research with two user groups (adults enrolled in literacy classes and in an essential skills program), and contrast the educational impact and attitudes toward such technology between these. Our conclusions present the lessons learnt from our evaluations and the impact of the studies' 
specific challenges on the outcome and uptake of such mobile assistive technologies in providing practical support to low-literacy adults in conjunction with literacy and essential skills training.

Keywords: Mobile computing, educational interfaces, mobile learning, assistive technology, evaluation methodology.

\section{Introduction}

Basic literacy skills are fundamental building blocks of education, yet for a very large number of adults, tasks such as understanding everyday items including bus schedules, food labels, news articles, or medical information is a challenge. In 2000, nearly $25 \%$ of adults (aged 16 to 65 ) in the world's richest countries were considered functionally illiterate [33]. In Canada, 50\% of adults are considered to have low literacy skills [1]. As the literacy-based demands of today's society grow, those adults with low literacy skills are becoming increasingly limited in their ability to understand, use, find, produce and benefit from textual information required in daily activities at home, at work and in the community, with significant personal and economy-wide consequences. Although many community-based organizations offer resources and support to such adults, current programs have difficulty reaching and retaining those who would benefit most.

The portability and affordability of mobile devices offers a realistic opportunity to provide novel, context-sensitive literacy and essential skills resources both within and outside community programs. We have thus decided to investigate how adults enrolled in such programs can benefit from a mobile assistive technology that supports experiential learning, and furthermore investigate how the perceived usefulness and ease of use of such technology influences students' independence and confidence, as well as their motivation for improvement. By actively involving adult literacy students and literacy facilitators in a series of focus groups and participatory design sessions, we developed ALEX@ , a mobile $\underline{\text { Adult }}$ Literacy support app $\bar{\equiv}$ on for EXperiential learning and essential skills acquisition (Figure 1 and detailed in [23]). ALEX@ is designed to facilitate, in a manner sympathetic to the needs of functionally illiterate adults, a series of language-related and workplace skills support tools. Our ultimate goal for the system is that it will provide practical support to functionally illiterate adults in their daily life experiences and allow such adults to push beyond their comfort zones to become increasingly literate and independent.

\section{Background}

Although workplace training programs and adult literacy classes both appear sound alternatives to traditional educational institutions, there are major barriers for adult learners. Work, lack of financial resources, childcare, and transportation often prevent potential learners from taking part in and benefiting from such programs [2]. Barriers to retention in training programs include the inflexibility of program delivery that restricts the participant's ability to simultaneously continue working [34]. 
Despite considerable investment of time, effort and resources, there is little improvement in adult literacy [34]. According to a Canadian Council on Learning Report, projections of literacy rates reflect a virtually unchanged literacy level in Canada at $47 \%$ of adults by 2031 . This indicates a need to investigate alternative approaches to tackling adult literacy issues - namely, the use of pervasive and ubiquitous computing to support experiential language learning amongst functionally illiterate populations.

The majority of mobile language literacy research focuses on second language learning [19] such as: delivering vocabulary lessons and quizzes to the learner's mobile phone [35] capturing and sharing how learners practice language skills outside the classroom [16], [28] and sharing location-specific knowledge with other learners [26]. Other examples of language software for mobile devices developed for learning English as a second language (ESL) include-PALLAS, which allows for personalization of learning resources [29] 스실 and colleagues [3] outline another ESL toolwhich offers grammar and exercise books/content on mobile devices, albeit without providing feedback to students. "Mobile Mazes" is another mobile application that provides reading mazes (much like "choose your own adventure" books) [36].

Other mobile systems for ESL training involve the extensive use of images, such as the SIGMA system [13], which presents five second user-generated movies related to a word while displaying the spelling, meaning, and pronunciation of the word. Likewise, Joseph and colleagues' PhotoStudy mobile phone system allows ESL students to collaboratively build a system of image/vocabulary flash cards [15], although the system lacks pronunciation support.

Mostmobile applications for assisting language learning are aimed at second-language learning [19], with significantly less research dedicated to functionally illiterate adults. One notable exception is Attewell and colleague's 2004 publication [4] on using mobile devices to deliver literacy building content to disengaged young adults. Most other solutions are in the form of desktop software, ranging from audio-visual interfaces [11] to applications adapting texts such as websites to low literacy level readers [38], [39]. However, solutions like this do not address the larger issue of literacy education, which is essential to improving low-literacy adults' work performance and quality of life [8].

Studies examining the use of mobile aids by marginalized users are currently carried out in developing countries, and, to a lesser extent, in the developed world. Working with marginalized users presents researchers with a broad set of unique challenges for data collection, which can impact technology use as well as research methodology [18]. Users in developing countries who are less technologically literate conflate the hardware with the software in designs and discussions [22]. Such participants find abstract scenarios and introductions difficult [12], leading to problems with participatory designs and paper prototypes [6], [12], [21]. There are also challenges particular to users' social circumstances (as demonstrated by studies in North America with homeless populations [17]) or to where data collection takes place - researchers often have to collect data in public spaces within the community instead of in controlled laboratory environments [12]. 
Low text and computer literacy are also challenges for technology design [14]. Often marginalized users have low literacy, resulting in a lack of confidence in searching for or finding textual information [30], which can make traditional data collection methods, such as a structured questionnaires, difficult for researchers to administer. Lack of experience in formal information structures can also affect the uptake and evaluation of technologies based on these structures [20]. Furthermore, such users mainly rely on information from oral sources, and may feel like they are too old to learn and that technology is not useful in their everyday lives [27].

Recent developments in mobile technologies need to be investigated in order to provide new learning environments for literacy learning by extending beyond traditional learning paradigms and embracing the notion of experiential learning. In our research experiential learning is acquired through the performance of everyday life activities that focuses on the learning process of the individual [19].

\section{A Mobile Approach to Adult Literacy}

We are located in the Canadian province with the largest percentage of adults with low literacy levels. As such, for the ALEX project we have partnered with Government of New Brunswick's Community Adult Learning Services (CALS) to address the adult literacy and essential skills problem in this province. CALS focuses on adult learning services, including computer, literacy, and workplace training. The project's goal is to explore how adults enrolled in such programs can benefit from a mobile assistive technology supporting experiential learning, and furthermore investigate how the perceived usefulness and ease of use of such technology influences students' independence, confidence, and motivation for literacy skills improvement. We also aimed to empower learners outside of the classroom, to adapt to and meet the diverse needs of different learners and to offer an additional tool for teachers to enhance the classroom experience.

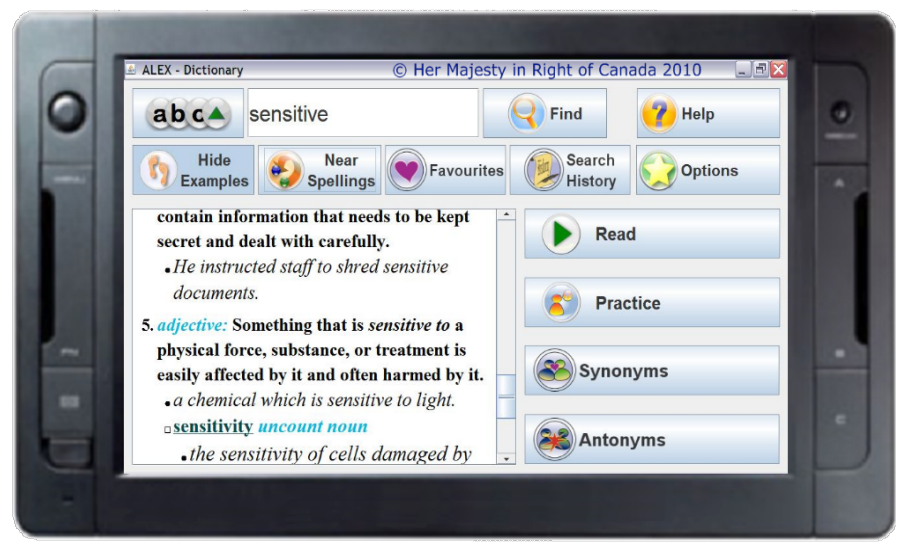

Figure 1: The ALEX® application, running on a 7-inch tablet.

\section{Implementation}

ALEX® was designed through a participatory approach involving teachers and students from adult literacy classes, and following guidelines for inclusive design of mobile assistive tools [19], as described in this Section and detailed in [23] and [24]. T) $\equiv$ 
language-based tools (Figure 1), it aims to not only support classroom exercises and homework but also other non-curricula activities such as reading the newspaper, interpreting a safety notice posted in a public space, writing a letter, and filling-in documents at home.

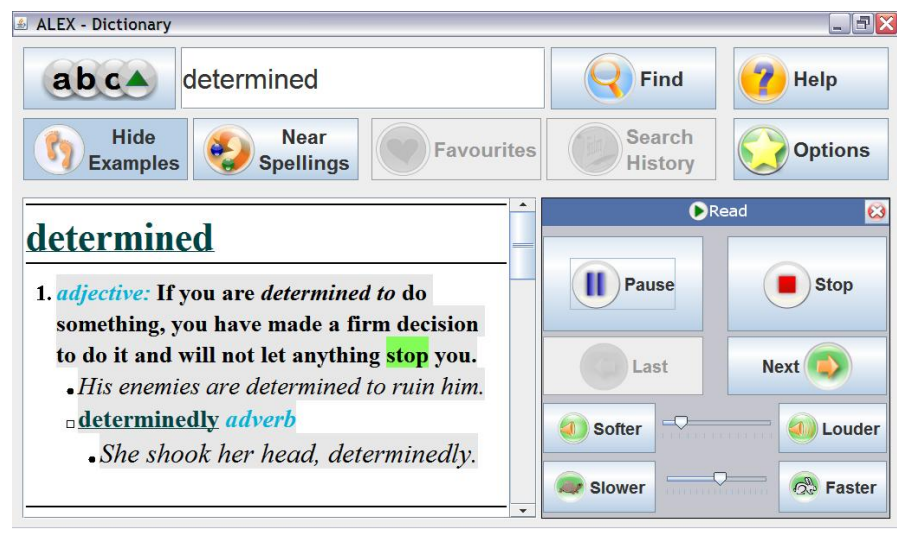

Figure 2: Dictionary look-up with audio spelling.

The main feature of ALEX@ is the look-up of definitions in the installed dictionary (Figure 2), with word usage examples shown under each definition ${ }^{1}$ (which users can turn off or on.) User-adjustable text-to-speech assists learners in reading definitions or in having the button labels being read to users. Words can be entered through either a QWERTY or a vowel-aligned virtual keyboard (Figure 3), with assistance provided by a spelling suggestion function (Figure 4). A persistent favourites-type list (Figure 5) and non-persistent history list implement web browsers metaphors that most users are familiar with. Other resources of the installed electronic dictionaries can also be accessed, such as synonyms or antonyms (Figure 6). An intuitive wizard-like process guides learners through a pronunciation practice figure (Figure 7) that provides positive reinforcement messages ("you did great", "try again, you're still doing great", etc.), based on the correctness of their pronunciations as determined by a customized speech recognition system.

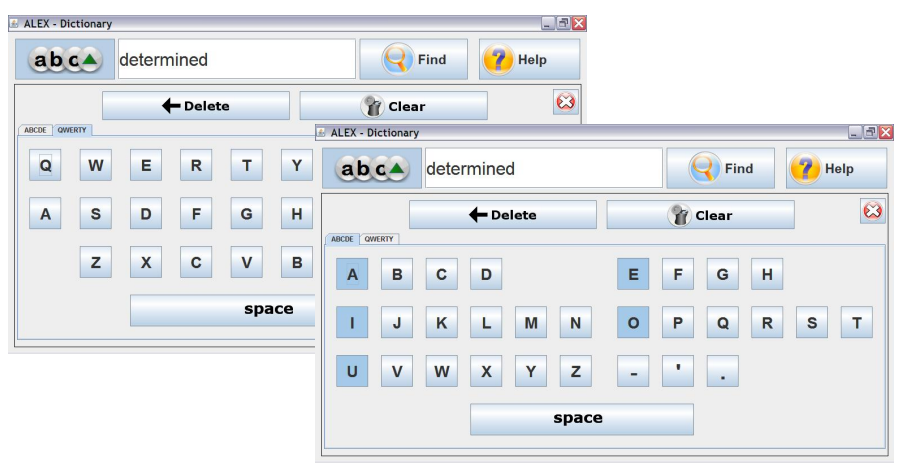

Figure 3: Alphabetic and QWERTY text input.

1 For the study presented later in this paper, the "Collins Cobuild Advanced Dictionary of American English" 1st Edition (C) HarperCollins Publishers 2007, and the "Collins Gem Thesaurus" 6th Edition (C) HarperCollins Publishers 2009 were embedded in ALEX(C), although any XML-based dictionaries can be uploaded. 


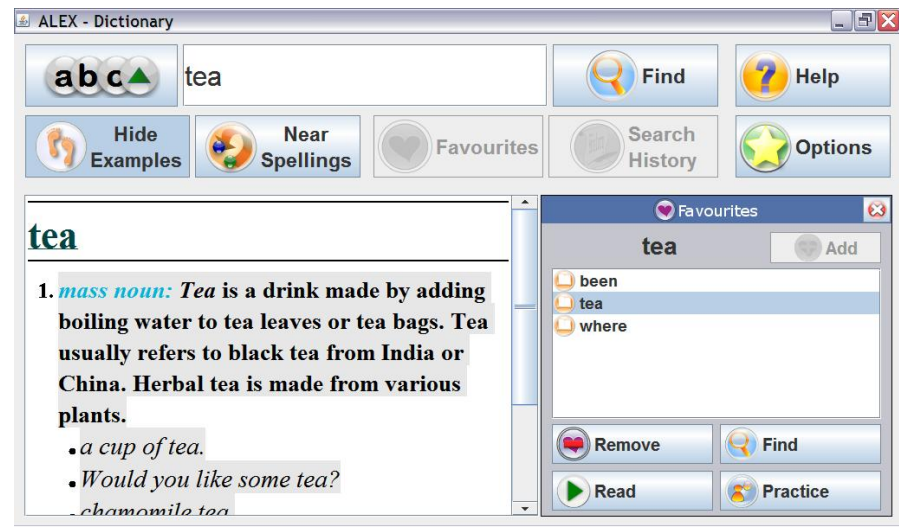

Figure 4: User-defined word lists.

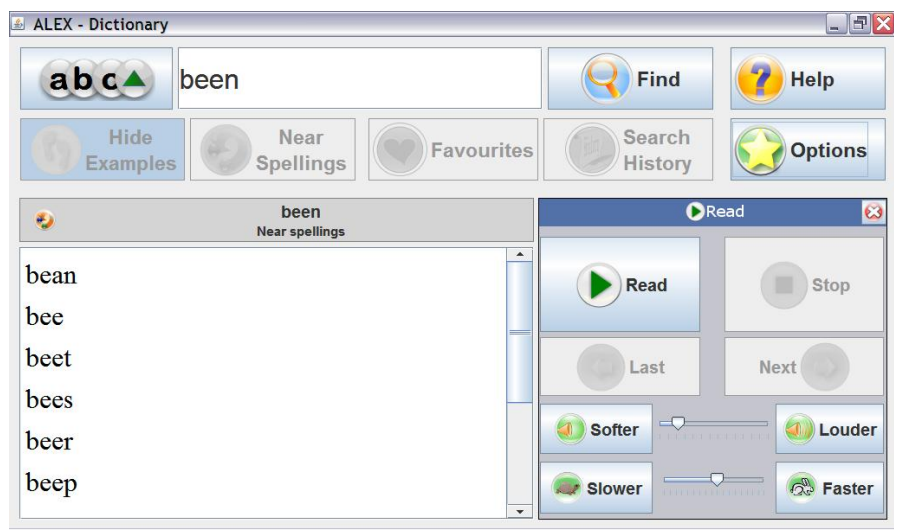

Figure 5: Suggestions of similarly-spelled words.

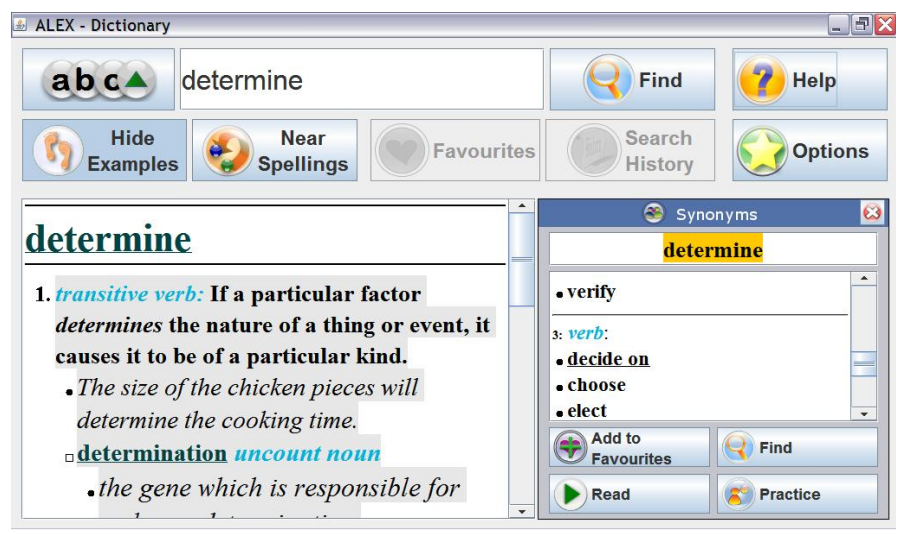

Figure 6: Language tools (e.g. synonyms).

\section{Overview of our two studies}

We conducted the evaluation of ALEXC through two studies. The first study lasted ten months within an adult literacy program dedicated to improving general reading and writing abilities. A second study was conducted over four months within an apprenticeship program centered on basic science skills for apprentices who do not possess a high school degree and are thus not eligible to enroll in trade-specific certification programs. 


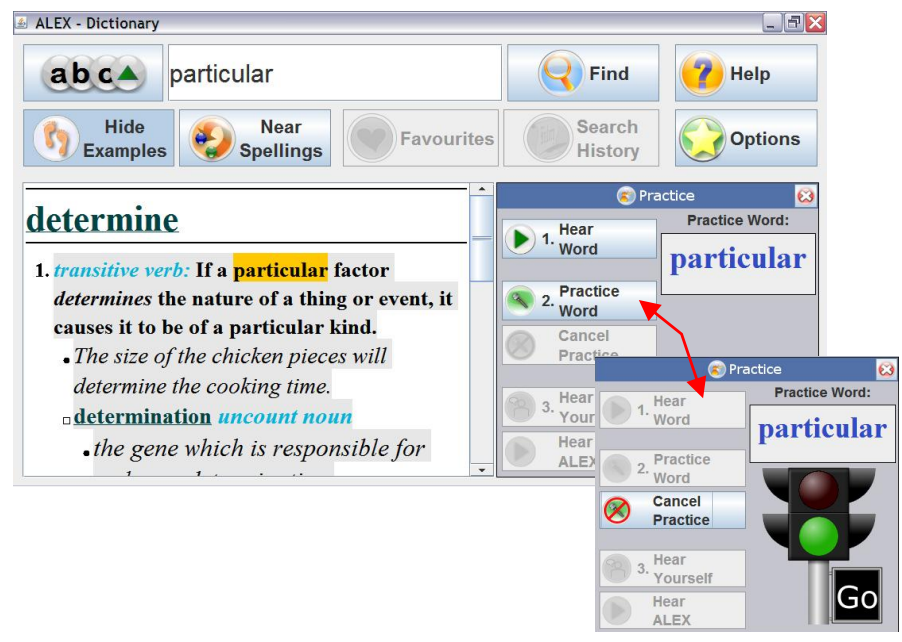

Figure 7: Pronunciation practice.

The unique characteristics of our user groups and settings (mainly, low-literacy adults in informal educational environments) presented significant challenges in determining appropriate evaluation methods. After observing similar classes-20 conducting participatory design sessions and interviews with teachers, we determined that a long-term exploratory study is the only suitable evaluation method. For this, each participant received one device running ALEX $\odot$, to be used both in and outside the classroom. Data was collected several instruments: discussions with

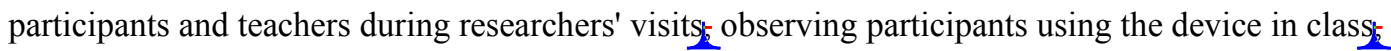
questionnaires administered by researchers as semi-structured interviews返 and teacher interviews at the end of the study. For assessing usage outside the classroom, we had to rely on participants' own verbal accounts, due to the impracticality of alternatives such as diaries given the participants' low literacy skills and due to ethical and legal limitations of the agreement with our partner.

For both studies, participation was entirely voluntary and teachers did not pressure students to enroll. Sign-up for these classes not mandatory, and all learners were of legal age. Eleven participants in two classes (six in a morning class and five in an evening one, each with a different teacher) were enrolled in the literacy study. Seven students and 2 teachers in one class were enrolled in the apprenticeship study.

No hypotheses were formulated before the evaluations - both studies were exploratory, aimed to discover how this particular technology is adopted and the outcomes of using it in conjunction the two programs. However, the study protocol, instruments, and data collection were guided by principles of the Technology Acceptance Model (TAM) [9], which postulates that perceived usefulness and ease of use are the main reasons for successful adoption. To avoid the risk of post-hoc interpretation specific to qualitative studies [32], an analyst and developer associated with the project acted as impartial observers during all data collection (observations, discussions, interviews with students and teachers). Care was also taken to transcribe factual information and not interpret the observations during note taking. The transcripts were analyzed by employing clustering techniques [32] to identify the main themes and outcomes of the study. 


\section{Adult literacy study}

\section{Methodology}

Potential participants received one tablet device running ALEX( , and were instructed on its use ane-on-one hour-long session with the researcher. After a review period of approximately one week, participants decided if they wanted to continue with the study. Researchers explained the study objectives and reviewed the consent forms, which participants had several days to review on their own, with family members, or with their teachers. Teachers were careful to not pressure the participants to enroll in the study. Independent of study completion, each participant received a $\$ 50$ voucher as compensation.

The eleven participants used ALEX $\odot$ in two distinct settings. In observed how students use the device for class exercises, and engaged with students in discussion about the application. Participants kept their devices for the entire duration of the study, and were encouraged to use it in the same way as their support materials (e.g.dictionaries) for homework or recreational reading, and explore ways to use ALEX in other situations.

\section{Challenges}

Most of the challenges encountered in conducting a comprehensive evaluation of our proposed solution are intrinsically tied to unique characteristics of our user base, including the student's_ literacy level $\mathbb{R}_{\mathbb{R}}$ the class format and technology acceptance.

\section{Literacy level}

A typical learner has completed some years of formal schooling, usually up to the end of middle school, and works part- or full-time in a non-professional position. They are only able to $\equiv$ rry out simple reading and writing tasks (e.g. ${ }_{\lambda}$ newspaper reading, short letter writing). To compensate for this, the researchers engaged in extensive on-going discussions with the teachers, followed by an in-depth interview with them at the end of the study. This provided us with teachers' perspective on the use and acceptance of the technology that complemented participants' narratives and gave us indirect access to students' daily interactions with teachers.

\section{三lass format}

The adult literacy sessions are usually conducted in an informal setting resembling oneon-one tutoring. A typical class consists of several adult learners, with one teacher per class, who guides students toward their individual goals at their own pace. Students work independently on their assigned subject which can be different from other students, making use of the provided materials (e.g. textbooks, dictionaries). The teacher moves between tables, assisting students or answering questions. Students chat among themselves, occasionally helping each other with their work. There is no formal evaluation of academic progress. 


\section{Technology Acceptance}

As expected, most of the participants, particularly the older ones, were reluctant to use the technology or to fully explore its features at the beginning; a reluctance that subsided as the study progressed. Several minor issues that initially created anxiety for participants (e.g_accidental changes of settings in our application and in the general operating system) were quickly addressed by researchers, providing participants with the reassurance that technology, especially software, is not threatening and can often be repaired.

Another challenge was the loan status of the devices - some users were overly cautious with the devices. For example, one potential participant withdrew from the study after one week for fear of losing the device. In other cases we had to replace devices or chargers due to physical damage, while some participants went to great efforts to conceal accidents, despite our promises of no consequences for broken devices (e.g ג $_{\text {s }}$ sper-glue inside a device that was probably dropped).

Overall we have discovered that ALEX(C) eased the participants' fears and facilitated their use of the technology. After the initial reluctance, all students were able to fully use the system.

The system "is easy to use, it is not scary or complicated" [Teacher T1]

This finding complements the main reason for adoption of the system - its perceived usefulness.

\section{Outcomes}

The evidence from our longitudinal study confirmed that both usability and usefulness were important factors in the acceptance of ALEX(C) and in its positive impact. We describe here the findings of our study, grouped according to the identified themes: perceived usefulness for homework 造perceived ease of use $e_{2}$ perceived value in terms of time saved and changes in participants' attitudes and independence.

\section{Usefulness for Homework}

All participants appreciated the helpfulness of ALEX@ for homework - four students even asked us if they could purchase the devices. Both teachers indicated that students employed it mainly in relation with exercises in the literacy curriculum (word look-up, synonyms, antonyms). According to teacher T1, students often ask for help in the classroom, but outside they do not have any resources, thus ALEX® helped the most at home.

Among other features students found useful for homework were Near Spelling and Read. Participant P3 indicated that hearing the pronunciation of new or difficult words helps her remember how to spell that word. Teacher T1 indicated that most of her students had difficulty writing new words, thus typing in their best guess and using the suggested spelling helped.

The example sentences included in the dictionary were also valuable. Participant P1 indicated that this was useful for understanding the meaning of words when the definition was too complicated. Participant P2 used the example sentences to learn new words, which she found helpful for writing essays. 


\section{Ease of use}

As our users had a wide range of backgrounds with respect to technology use, we were initially concerned that a tablet device may pose usability challenges. However, our approach of involving teachers at early stages of the design and following guidelines of inclusive design for mobile applications resulted in the device being perceived as easy to use:

"it was easier to use than a paper dictionary" [all participants and teachers]

Participant P5 found ALEX@ less confusing than a traditional dictionary. According to teacher T2, P5 forgot how to properly use a traditional dictionary, but found our system intuitive. Furthermore, this motivated him to look up even more words.

Teacher $\mathrm{T} 1$ indicated that ease of use is the main advantage of using ALEX@ over a paper dictionary, since students are more likely to look up or double-check words when they are in doubt about their use or meaning, which usually does not happen with paper dictionaries.

Participant P1 indicated that the Near Spelling function contributes to the ease of use. She often misspells words, and with a paper dictionary finding the definition of a word by guessing its spelling is difficult.

Participant P3 mentioned that it is easier to come back to a word she has difficulty with using ALEX® compared to a paper dictionary.

\section{Saved Time}

Users found ALEX@ provides easier and much faster access to definitions, compared to traditional dictionaries, resulting in significant time saved. Participant P3 indicated that she has difficulty finishing her homework between a full-time job and childcare, but with ALEX she can finally complete her homework. Other students have mentioned similar benefits:

"I can do my exercises like, two times faster now" [P1]

ALEX( ) was also appreciated as a time-saver by the teachers. Both have indicated that students ask less for help with simple tasks, and instead call them only for more complicated tasks, such as understanding the meaning of a complete sentence.

\section{Helped gain independence}

One of our project's goals was increasing adult learners' independence by assisting with everyday tasks, as well as encouraging the use of literacy resource $\bar{\equiv}$ ond schoolwork. Indeed, we have found that ALEX $\odot$ allows low-literacy adults to perform thore independently.

Participant P5 has always felt embarrassed by his lack of literacy skills. He likes reading the newspaper but was frustrated that he doesn't fully understand all of the articles. With a mobile language assistant, he started reading the newspaper and doing his homework at a coffee shop. He felt socially accepted, since the shop is typically frequented by college students who use laptops and mobile devices while studying there. Teacher $\mathrm{T} 2$ noted:

"P5 would never buy a dictionary or want to be seen with one. I was surprised seeing him using ALEX at the coffee shop - he even used the [audio spelling] with his headset on -I guess he felt it's cool to have them like all the young students." 
Teacher T1 mentioned that students also used the devices to write their shopping lists P1, P2, and P3 all indicated that it would be even more helpful it they could write entire recipes posted in the store and get definitions for the words.

Participant $\mathrm{P} 3$ also reported being interested in politics and finds ALEX( ) helps with the difficult words in the newspaper. Similarly, others mentioned using ALEX $\odot$ when reading books at home.

Both teachers reported on usage outside prescribed academic activities:

"P4 uses it for everything!" [T2]

From students' narratives, we have also identified situations where other technologies would have been more convenient, yet for this particular group ease of use and becoming accustomed to ALEX $\odot$ prevailed. For example, participant P1 mentioned using Near Spelling when on instant messaging, while participant $\mathrm{P} 3$ used the same spelling feature for e-mails.

Participant P3 also reported using ALEX) in social situations. Partieularly, when a debate started during dinner about a word, she claimed to know its meaning, and then used ALEX to quell her guests' disbelief:

"It was cool to show off to my friends" [P3]

\section{Increased confidence and motivation to learn}

One of the most important findings of our study was the increase in learners' confidence and motivation to learn. This was both confirmed by teachers and self-reported by participants.

Participant P3 mentioned that she is now more confident that her homework is correct:

"I see the word coming up on the screen and I know I got it right"

Participant P4 indicated that she likes to increase her knowledge in general, and ALEX® provided her with encouragement, at least for the English language.

Both teachers reported that such a system provides an incentive for students to learn:

“... reignite the spark when things are getting boring” [ $[\mathrm{T} 2]$

“... made learning fun and was a real confidence-booster” [T1]

"It was less hassle and less boring than a regular dictionary" [T2]

Teacher T1 also-mentioned the case of a student (not a participant in the study) who was preparing for his GED (high school equivalence) exams. He doubted his ability to study for these, but after using the device in the classroom he became confident in his abilities.

Teacher $\mathrm{T} 1$ also reported that students realized the importance of dictionaries, which they didn't see before and thus lacked motivation to use them. Furthermore, the occasional failure of ALEX@ to find the meaning of a word prompted discussions about the inherent limitations of dictionaries and comparisons with the paper dictionaries in the classroom - teacher T1 believes that this increased students' interest in the learning process. 


\section{Unexpected findings}

\section{Building a personal relationship with the technology}

When speaking to researchers some students phrased their statements as if ALEX $\odot$ was a person (perhaps influenced by its name/acronym). Students also attributed it human-like characteristiq- during some group exercises, they drew on the whiteboard a human shape named ALEX and left the adjacent space on the table empty. This was projected outside the classroom too - participant P2 received a pocket translator as a gift and named it "baby ALEX".

\section{Sharing the system}

Participants shared their devices with friends and family. All participants that had schoolage children reported lending them the devices for homework. P2 mentioned sharing the device with her sister to help her improve her English. P3 reported that her 8-grade son found it easy to use and helpful for essays, while her daughter even demanded she purchase a device for her.

"[My son] finally found something he is interested in ... he doesn't care for books at all" $[\mathrm{P} 3]$

P6 even wanted to share ALEX $\odot$ with her preschool-aged son to teach him new words, but was fearful of device damage.

Participants also shared devices in the classroom with students not enrolled in the study. Participants P1 and P2 reported introducing the technology to other students and explaining how to use it:

"I don't even have to explain how to use it, [P1 and P2] are doing it. This is a good learning experience, when one student explains something to another." [T11

\section{Pride in taking part in the study}

We found that participants understood the process of validating technology through evaluations with real users. As the study progressed, they became less shy in providing feedback and criticism - teacher T1 reported that students were anxiously waiting for researchers' visits so they gan report on the system's usage. They took pride in being part of the study by identifying issues and suggesting solutions. Some even spent time looking for problems, despite often conflating hardware issues and the application's design. Participants liked the fact that someone was listening to them in a field that they had not contributed to before, and felt that it was important to tell us about problems that they found - teacher $\mathrm{T} 2$ reported that students felt that someone was listening to their needs and struggles.

\section{Unforeseen factors}

Demographic factors, such as age and employment opportunities played an important part in influencing learners' motivation for improving their education. Anecdotal evidence collected in this study indicates that the younger, more employable, learners would use such an application if it 
was offered in a different format, such as a smartphone app. As teacher T2 noted, these learners already owned a smartphone - some despite not having the required financial means.

Both teachers agreed that such a mobile language assistant is most useful for adult learners that are "in the middle" with respect to their literacy skills, and that for advanced students the benefits stems mostly from convenience. The exception was participant P1 who has a personal ambition to eventually study English literature at the university. However, both teachers reported that students at lower levels of literacy, and immigrant students struggling with English were intensive users of ALEXC.

\section{Potential Applications}

Amongst the several improvements suggested by teachers and students, exercises in the form of games was the most recommended. However, regular exercises would not be welcome by students, and that the "fun" aspect would disappear:

"Students would just start hating it, like they hate doing homework." [T2]

Almost all participants have indicated they would like to see some form of basic math help included, and both teachers confirmed that all students struggle with math.

Participant P2 indicated that she would like a bilingual translator integrated, to help her understand some complex words. Other students mentioned the need for more complex language support, ranging from grammatical help (e.g. processing (e.g. an entire sentence's meaning):

"I want to get the same help as [our teacher] gives us." [P3]

Students stated that they would like to see an "all-in-one" device, with ALEX@ integrated with common applications such as e-mail [P3, P4] or text editors for essay writing [P2]. Students also suggested integrating encyclopedic resources, such as geographical information, spelling of proper names, or as P4 suggested as helpful for her Canadian citizenship test, information on historical figures.

\section{Workplace essential skills study}

After evaluating the language assistant capabilities of ALEX $\odot$, we adjusted the design and implementation to support learning in the context of essential skills training. For this we partnered with SkillPlan, a major Canadian publisher of science books for construction apprenticeship curriculum. We have extended ALEX@ to include general science and math references (Figures 8, 9, and 10). The added features assist users in navigating the more complex structure of science materials: calculator (both standard and scientific) 迤 table of contents板 bookmarkss and-an annotation capabilities that allows users to type in short notes anchored to any part of the displayed material (e.g. . $_{\text {text, }}$ image, formula). 


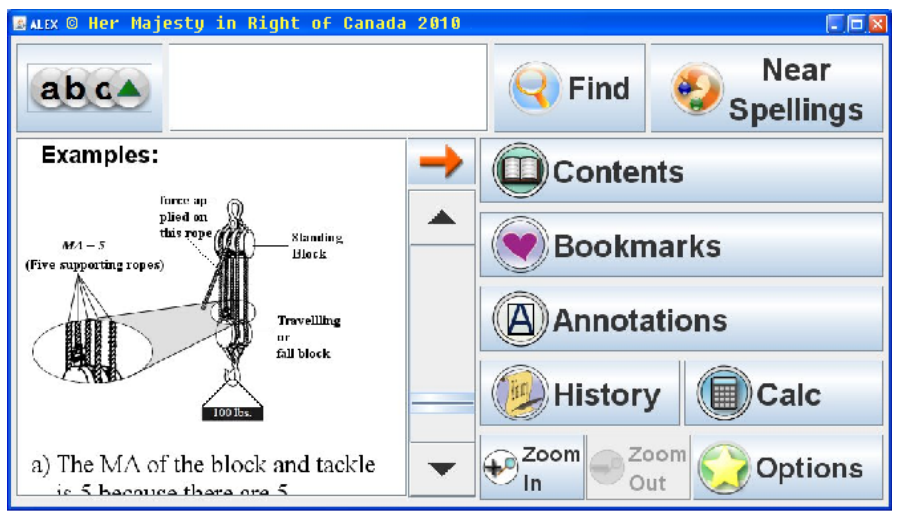

Figure 8: Interface and functionality adapted for use in workplace essential skills training.

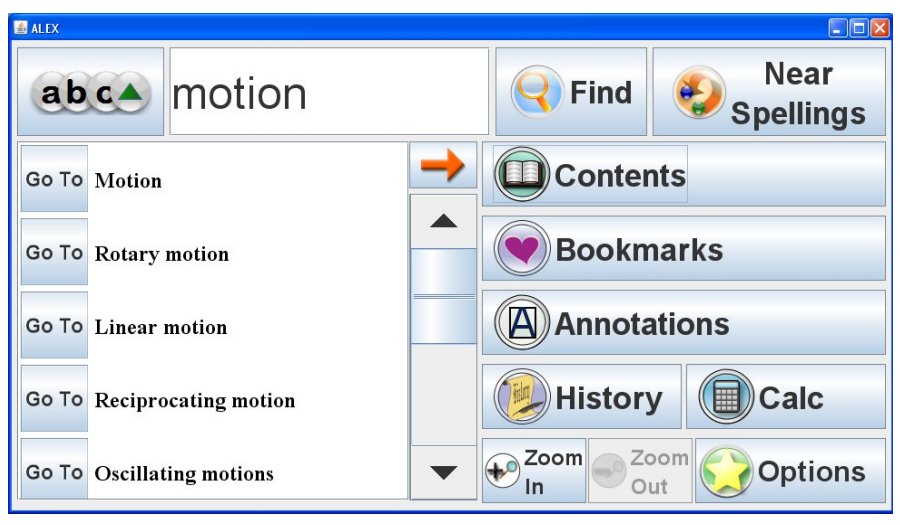

Figure 9: Extension of the look-up concept to include reference materials.

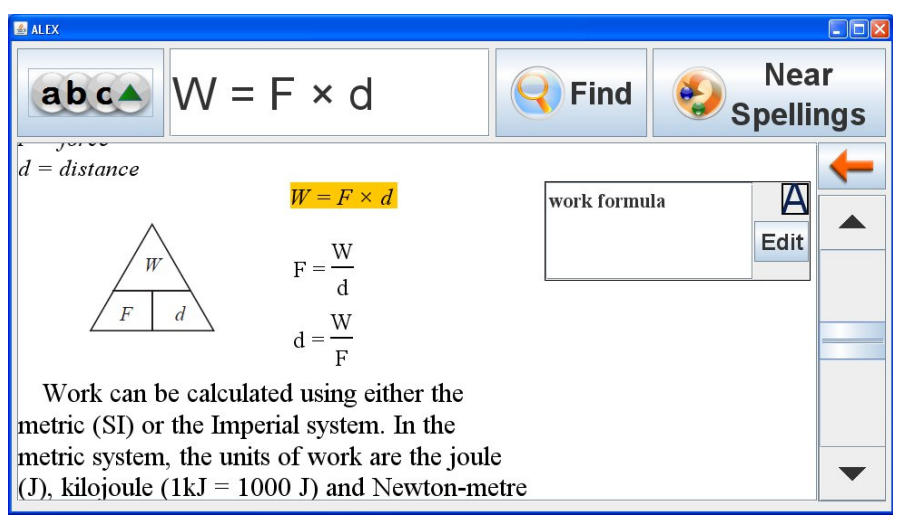

Figure 10: Fullscreen view of a textbook section with usercreated annotations on the side. 


\section{Apprenticeship classes}

CALS' apprenticeship classes are part of the Workplace Essential Skills (WES) program designed to support adults looking to acquire the skills needed for work or learning, as well as provide the credentials to register as apprentices (after which they can work toward certification and better employment). A typical learner is aged 20 to late 30 s and working without the required high school or equivalent degree.

The apprenticeship classes are also conducted in an informal setting. However, in contrast with the literacy classes, the learning materials are more structured, and students are encouraged to work independently on photocopied exercises at their own pace, with the instructor intervening only when students need specific help.

\section{Evaluation}

We followed an evaluation approach similar to that conducted within the literacy classes. The evaluation consisted of a pre-pilot phase and a study where initial design assumptions were verified, in a suitable class as identified together with the WES coordinators. The selected class had an enrollment of nine students, and one teacher (with a substitute teacher filling in occasionally $)^{2}$.

Our design assumptions were informally validated through several sessions with the teacher of the selected class, and through a two-hour session with two graduates of a similar class. Minor changes were implemented based on feedback, such as the addition of text size control buttons and the ability to switch the display from split between textbook and navigation to fullscreen textbook view.

The evaluation protocol was similar to the literacy study, with devices being lent to learners. Differences included number of participants (seven of the nine students signing up, with five completing the study), duration and frequency of classes (three months, twice-a-week classes of two hours each), classroom format (less group interaction), and researchers' presence (once a week due to location in a different city) and interaction with participants (15 minutes at the end of class). Participation was voluntary and financially compensated with a $\$ 50$ voucher. At the end of the study guided interviews were conducted with each participant.

Since scheduling constraints did not permit a follow-up longitudinal study, we complemented the collected data with an informal follow-up participatory design session, that was compensated with a $\$ 20$ gift card. Three students participated in a two-hour brainstorming on extending the design and functionality of ALEX to better provide support for on-the-job training.

\section{Findings}

Although the apprenticeship study was comparatively shorter than the literacy longitudinal study, we were able to identify several emerging themes related to technology

2 We will refer to the participants in the apprenticeship study by using the A- prefix in front of the participant ID (e.g. A-P1). Similarly, the teachers will be referred to as A-T1(the main teacher) and A-T2 (the substitute teacher). 
acceptance and adoption. We summarize these here, and elaborate more on the challenges and implications for design in the next section.

\section{Attitudes toward social acceptability}

Our assumptions that ALEX $\odot$ bridges the gap between the knowledge acquired in class and its practical application on the jobsite were challenged during the study. Some participants expressed doubts about using ALEX@ on a worksite: from an employer policy perspective (e.g $\mathrm{g}_{\text {no }}$ cellphones), being worried about damage, or simply speculating about supervisors being suspicious about them (-in contrast to the literacy study, in which learners saw the device as a vehicle for increased social acceptance). However, participants would use the device on the worksite if its practicality was evident (e.g. , $_{\text {it }}$ had building codes and calculators) and understood by supervisors:

"I would take it with me since it's a time-saver, but maybe leave it in the truck-I guess it's up to the boss if he gets it that we're not just goofing around with it." [A-P8]

\section{Attitudes toward learning}

In contrast to the adult literacy participants, the learners in the essential skills had a dismissive attitude toward education. This was not unexpected, as teacher T1 from the literacy program indicated that lack of literacy skills is often perceived as a social stigma, while lack of numerical and essential skills is mostly not:

"papersmart don't mean nothing at the jobsite." [A-P2]

Some participants stated that math exercises have "nothing to do with carpentry" [A-P2, A-P4], while A-P4 stated that he "can't see how the math and practical related", despite the numerous examples in the ALEX( $)$ materials or given by the teacher illustrating the connection between math and real-world problems, and further mentioned that not even his boss received a complete formal education.

\section{Perception of on-device textbook suitability and usefulness}

Students perceived books to be too theoretical, and they've projected this onto the device as well (possibly also due to the mismatch between the textbooks uploaded into ALEX@) and the class exercises that changed over time). Their expectation was of a highly interactive handyman's pocket guide, not that of easy-to-access textbooks (despite the textbooks' practical focus). This was compounded by familiarity with smartphones - A-P5 noted that he doesn't use the home computer for more than browsing online stores, but he'd like the idea of ALEX® on an iPod ${ }^{3}$.

The feedback from the follow-up design session confirmed the need for-a full smartphone-like interaction. However, participants indicated that it must meet certain physical requirements, such as ruggedness for using in a truck while planning for that day's work - "so people are less afraid to use it" [A-P4].

3 We have since ported ALEX@ to both iOS and Android. 
The teacher noted that the older students needed a longer time to get comfortable with the technology, but that eventually they all got accustomed. The most notable exception was participant A-P5, who, despite being in his early 30s, was dismissive of the technology:

"It's a generation thing - ALEX is for the younger generation. I am too old for this. The kids use computers all day, they will be able to use something like ALEX.”[A-P5]

\section{Challenges and recommendations for conducting such research}

Several significant factors influenced the design and proeeding of the two studies, often representing departures from more "traditional" evaluation methods. We describe here some of the most challenging factors as well as the approaches we took to ensure the collection of relevant data $^{4}$. We also make recommendations for the design of mobile applications in support of literacy and essential skills programs and for conducting future research with these particular user groups.

\section{Literacy Levels}

Both the literacy and apprenticeship students share similar educational backgrounds some years of (incomplete) schooling and the ability to carry out simple reading and writing tasks. Most of them work in non-professional jobs or in positions not requiring a trade certificate. The literacy and essential skills levels of adult learners are assessed directly by teachers during the enrollment interviews using internally-developed guidelines.

The participants' literacy levels make it difficult for researchers to conduct rigorous, structured data collection. Even questionnaires phrased at appropriate literacy levels did not elicit meaningful answers. Instead, most of the data was collected either through direct, individual interactions with users during the longitudinal study, or prompted by the verbal administration of the questionnaire at the end of the study with questions often rephrased as a personal story or example. This approach proved more successful - participants' often-unsolicited narratives and direct observations provided researchers with an intimate understanding of both participants' struggles and needs. As such, we recommend avoiding written surveys and highly structured interviews in favour of informal conversation-styled interviews that are tailored to the individual.

\section{Researcher Bias}

The particular conditions surrounding the two studies, particularly the adult literacy study, posed challenges in maintaining researchers' impartial, non-intervening, and unbiased position. Students quickly became familiar with the researcher - a positive consequence, resulting in unreserved feedback to researchers, but also an expectation of researchers to become more

4 An extensive analysis of these factors and detailed design recommendations can be found in [25]. 
involved in the class. For example, even during the review week prior to signing the consent forms, some users started providing feedback and expected us to take notes.

To maintain impartial data collection two researchers were on site most of the times, resulting in a significant amount and depth of data. In fact, Teacher 1 from the literacy study confirmed students' eagerness to provide feedback to researchers. Teacher 2 mentioned that the literacy students felt less marginalized by contributing to something that would help others like them.

We suggest that bonding with subjects is not only inevitable during long-term studies, but is desirable. We believe the resulting increase in trust and empathy outweighs the risk of researcher bias. To reduce the impact on the impartiality of data analysis, we recommend having at least two researchers present during observational studies and to include researcher interactions with subjects as a data source. We also recommend the data analysis be conducted by an additional researcher.

\section{Participant Attendance}

Irregular attendance, due to most students juggling work, family and course commitments, was an issue during both studies, making collecting feedback, observing usage, upgrading the system, and even scheduling end-of-study interviews difficult tasks for researchers. Unexpected withdrawals or long-term absences were not uncommon. Outside factors (weather, lack of vehicle, unexpected work commitments) also affected attendance.

In the literacy classes, we noticed that attendance was more frequent for older participants than for younger ones (with the exception of P2 - a younger immigrant student). Of the older learners, the participant with infrequent attendance (participant P5) withdrew from the program when he left town for work. This distribution specific to the literacy program was explained by teacher $\mathrm{T} 1$ through older learners' awareness of the positive impact that education has for employment, with younger learners more willing to trade education for immediate employment and having difficulties attending classes while working shift jobs.

In the essential skills study nine students were initially enrolled in the course. Two dropped the course early on due to work commitments and of the remaining seven only five attended classes at all, with only one attending on a regular basis. The course instructor was keen to highlight that this was not a usual occurrence:

"Not sure ALEX@ was for this group, part of this because of attendance, which is not normally an issue, but it was for this group." [A-T1]

Attendance posed challenges to conducting the two studies as well, due to limited opportunities to consistently carry out class observations for students with infrequent attendance. Difficulties contacting these students also affected the closing interviews -6 of 11 students in the literacy study and 5 of 7 in the apprenticeship study were present at the studies' completion.

Irregular attendance may be an inconvenience for researchers but it is the reality of the domain being studied. As such, we recommend supplementing observational studies with interviews to reduce the potential bias towards students who attend most frequently. When 
researchers cannot attend all classes, we suggest implementing observation by proxy through structured interviews and ongoing dialogue with the teacher. Finally, it is practical to enable remote updates of the software, so that all participants can benefit from bug fixes.

\section{Participant Engagement}

Overall, we have found that participants understood the process of validating technology through evaluations with real users. Once the study progressed and students became more comfortable with the researchers, they became less shy in providing feedback and criticism (particularly the older literacy students).

Participants in the adult literacy study took pride in being part of the study by identifying issues and suggesting solutions. Some even spent time looking for such problems - unfortunately, it was difficult for the researcher to properly instruct the participants in identifying applicationspecific issues from hardware issues or from dictionary limitations.

In contrast, participants in the apprenticeship study were somewhat more disinterested in the study. This can be in part attributed to a more rigid class format, as well as to a dynamicallychanging curriculum which we did not anticipate. Another possible explanation, as detailed under "Findings" in the previous section, is students' rather dismissive attitude toward education in general and mathematics in particular, especially when their work experience enables them to gain employment.

As the apprenticeship study progressed we noticed a mismatch between what users expected from such technologies and what systems like ALEX@ deliver. To verify this, we conducted a participatory design session at the end of the study, after students became comfortable with the idea of mobile assistive technology supporting their learning needs. Three students participated, and provided good feedback about the device and their learning needs and expectations from mobile learning assistants. They were visibly more comfortable than during the classes, perhaps attributable to their expressed aversion formal teaching. A common theme emerging from their feedback was their expectation of job-specific aids (e.g. $\mathrm{g}_{\lambda}$ building codes or fillin interactive formulae for specific construction tasks). Despite the researchers' use of various props such as videos and leading the discussions toward learning goals, the students did not see the applicability of mobile technology in supporting learning and maintained their dismissive attitude toward education:

"The best way for me to learn is by taking something apart. That's how I've learnt the trade from my dad" [A-P5]

The status of the devices also impacted the-participant's_ willingness to use ALEXO. While in some cases, the status of having a new d interaction with others (as detailed in [2012_13]), Some participants were overly cautious with the devices, as highlighted by our observations in both studies.

Varying degrees of engagement with an academic study and technology itself is to be expected when carrying out studies with populations that are not composed of early adopters. As such, it is expected that some will reject the technology. We strongly discourage excluding such 
participants from the study. Therefore, we suggest taking the opportunity to learn more about nonusers and non-use (as in [31]) and find out what is of value to them. We recommend being prepared to tailor the level of researcher contact and participant involvement to that which is acceptable to the individual participant. Similarly, we need to acknowledge the limitations of technology's scope of influence - for those unmotivated to learn, consideration should be given to motivational and practical barriers to learning that could be addressed by technology.

Finally, if devices are being distributed as part of the study, we suggest budgeting for loss of devices and device repairs. Researchers may need to reassure participants that some accidental damage is to be expected and that they will not be charged for damage.

\section{Importance of Proxy Support}

Given the critical role that teachers had in the informal settings of the literacy classes, the successful adoption of technologies such as ALEX@ is dependent on teachers' support for such solutions - teachers act as proxies for students' acceptance of technology in the same way they support students' skills and knowledge acquisition. By involving teachers in the early design stages and in planning for the evaluation, they can integrate the technology in the classroom without negatively disrupting the class proceedings. Furthermore, our ease of access to, and interaction with users was immensely facilitated by teacher这 reinforcing the importance of proxy support when addressing the needs of marginalized user groups [21]. This was relevant not only in supporting the collection of meaningful data from low literacy users, but in establishing a relationship with researchers.

The apprenticeship classes were significantly less "hands-on" and more individuallyfocused, due to their science- and math-oriented curriculum. However, since students worked on different exercises at their own pace, it was difficult for them to know which topics would be covered next:

[ALEX® would be better] "If it was tailored to how the school was going to work. Here it was different - everyone was working in a different section and we jumped around a lot." [A-P4]

These aspects, combined with a much shorter and less-frequent schedule than the adult literacy classes, lead to significantly fewer interactions between researchers and students. The consequence for researchers was the inability to direct the study and guide the students in determining how ALEX@ can address their learning needs; instead, participants decided entirely en when and how to use the devices. This reinforced the importance of close interaction with researchers and active involvement of proxies in developing a supportive relationship with learners that is not limited to the usual rapports found in more traditional classrooms, in eliciting feedback from such user groups.

Based on these experiences, we recommend that, as with researchers, teachers' interactions with the students and the technology should be included as a data source for analysis. We also suggest conducting multiple studies in different instantiations of the same domain setting in order to fully understand the influence of proxy support and what helps or hinders technology uptake other than the technology itself. Researchers could also consider how such non- 
technological factors that influence uptake can be integrated into the system design, and address the question of how the technology should be promoted in non-study settings. If teacher and researcher involvement is central to uptake, this could indicate a limitation of the system's potential scope or a need to integrate the system into a curriculum change/teaching package.

\section{Summary of findings, challenges, and design recommendations}

Participants in the adult literacy course appreciated the helpfulness of our system for assistance with homework. In comparison, students in workplace essential skills, for the most part, did not use the device for homework. One of the major goals of our project was to increase adult learners' independence outside the classroom environment by assisting with everyday tasks, as well as encouraging the use of literacy resources beyond schoolwork or homework. Indeed, the analysis of semi-structured interviews with participants, recorded narratives, and interviews with teachers revealed that ALEXC allowed low-literacy adults to perform more independently and become more confident $\mathrm{f}^{\mathrm{f}}$ their literacy skills. Furthermore, we have gathered users' preferences for future developments of such assistive technologies, such as incorporating games, exercises and practice tests and integrating the system with other educational applications and learning environments.

Although shorter in duration and scope than the literacy study, the workplace essential study (WES) produced important preliminary information on the feasibility of technologies such as ALEXC for apprenticeship training. Due to a dynamically-changing curriculum and short duration of the study, WES participants did not report widespread use of the device outside of the classroom. However, students provided researchers with valuable feedback on the design, usability, and usefulness of mobile interfaces for technical and essential skills training. These findings carry significant implications for future developments and applications of ALEX® or similar technologies to other adult learning areas, such as numerical literacy or workplace and onthe-job training.

The challenges that we faced when developing and evaluating our literacy and essential

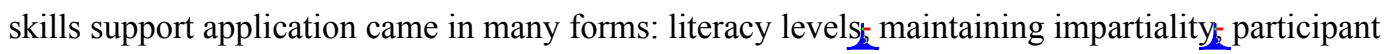
attendance participant engagement and the impact of proxy support. Two core themes have emerged from our subsequent recommendations. First is that of extending the scope of study design and data analysis from the student, technology, and context of use, to include the researcher, teacher, and impact of the study. The second theme is that of tailoring the study protocols and methods to the individual participants.

Both of these have the potential to impact the scientific validity of any study. Arguably, the first stands to increase the validity of such studies by explicitly including factors that are known to influence participant behaviour in the body of data being analyzed. However, the second demands methodological flexibility that seems to stand in opposition to the methodological rigour expected within the field of Human-Computer Interaction. As evidenced in the examples we have 
presented in this paper, we suggest that this aspect needs to be carefully considered when conducting evaluations of mobile assistive technologies for marginalized groups, and that no "easy-to-follow recipe" exists for how to address this theme.

\section{Conclusion}

The current literacy levels of working adults do not meet the demands of today's information-centric society. In Canada, existing literacy and workplace essential skills programs only reach a small number of those who would benefit from them [8]. As such, there is a need for novel approaches to adult literacy and skills training. In this paper we have presented a mobile application that enables adults to improve their language and essential skills outside the confines of available programs, and brings them closer to functional literacy and independence. The results of our two exploratory studies with low-literacy adults demonstrated that personal mobile devices are a viable technology for providing learning and functional support to such adults, and that many of the challenges they face are addressed by our mobile application designed to be used both in the classroom and in daily life. Mainly, we have shown that such a solution complements literacy programs by increasing users' motivation and interest in learning, and raising their confidence levels both in their education pursuits and in facing the challenges of their daily lives.

Additionally, through the workplace essential skills study, we have explored issues of acceptance and adoption of such technology and outlined design recommendations for tailoring mobile learning support applications to this particular user group.

We have also reflected on the particular challenges encountered by us, as researchers, in designing our application and conducting the two studies. While a significant body of research exists on similar issues, they are mainly confined to literacy in developing countries. We hope that by presenting the lessons learnt from our evaluations and the impact of the studies' specific challenges on the uptake and outcome of such mobile assistive technologies in providing practical support to low-literacy adults, we are encouraging more interaction, education, sociology, and language researchers to dedicate their efforts to addressing the significant yet largely ignored problem: functional literacy amongst working or employable adults in industrialized countries.

\section{References}

[1] ABC Canada. (2001) Who Wants to Learn? ABC Canada Literacy Foundation Report

[2] ABC Canada. (2005) Learning Living: First Results of the Adult Literacy and Life Skills (ALL) Survey

[3] Ally, M. et al. (2007) Use of mobile learning technology to train ESL adults. Proc. 6th Int. Conference on Mobile Learning, pp. 7-12

[4] Attewell, J. and Savill-Smith, C. (2004) Mobile learning and social inclusion: focusing on learners and learning. In J. Attewell and C. Savill-Smith (Eds.), Learning with Mobile Devices: Research and Development, pp. 3-12 
[5] Attewell, J. (2005) From research and development to mobile learning: Tools for education and training providers and their learners. Proc. mLearn, pp. 1-6

[6] Bidwell,, N.J., Reitmaier, T., Marden, G., Hansen, S. (2010). Designing with Mobile Digital Storytelling in Rural Africa. CHI 2010, ACM Press, 1593-1602.

[7] Canadian Council on Leaning. (2010) Report: The Future of Literacy in Canada's Largest Cities

[8] Conference Board of Canada. (2010) Adult Literacy Rate - Low-Level Skills. Education Report

[9] Davis, F.D. (1989) Perceived usefulness, perceived ease of use, and user acceptance of information technology. MIS Quarterly, vol. 13 no. 3, pp. 319-340

[10] De Jong, T. et al. (2010) A study of contextualized mobile information delivery for language learning. J. of Educational Technology \& Society, pp. 110-125

[11] Dhakhwa, S. et al. Sambad. (2007) Computer Interfaces for Non-literates. Proc. HCI International, pp. 721-730

[12] Gorman, T., Rose, E., Yaaqoubi, J., Boyer, A., Kolko, B. (2011). Adapting Usability Testing for Oral, Rural Users. Proc. ACM SIGCHI.

[13] Hasegawa, K. et al. (2008) Learning effects of self-made vocabulary learning materials. Proc. Conf. on Cognition and Exploratory Learning in Digital Age, pp. 153-159

[14] Jones, M., Thom, E., Bainsbridge, D., Frohlich, D. (2009). Mobility, Digital Libraries and a Rural Indian Village. JCDL '09, 309-312.

[15] Joseph, S. et al. (2005) PhotoStudy: Vocabulary learning and collaboration on fixed \& mobile devices. Proc. 3rd Int. Workshop on Wireless and Mobile Technologies in Education, pp. $206-210$

[16] Joseph, S. and Uther, M. (2008) Mobile language learning with multimedia and multi-modal interfaces. Proc. 4th Int. Workshop on Wireless, Mobile and Ubiquitous Technology in Education, pp. 124-128

[17] Le Dantec, C.A., Edwards, W.K. (2008). Designs on Dignity: Perceptions of Technology Among the Homeless. Proc. ACM SIGCHI (CHI'08), pp. 627-636.

[18] Leung, R., and Lumsden, J. (2008). Reflections on challenges and guidelines for designing and evaluating assistive mobile technologies. Handbook of Research on User Interface Design and Evaluation for Mobile Technology, J. Lumsden (Ed.), Idea Group Inc., pp. 609623.

[19] Lumsden, J. et al. (2010) ALEX: a mobile adult literacy experiential learning application. Int. J. Mobile Learning and Organisation, vol. 4, no. 2, pp. 172-191

[20] Marsden, G. (2003). Using HCI to Leverage Commendation Technology. Interactions. March - April 2003, 48-55.

[21] Marsden, G. (2008). New Users, New Paradigms, New Challenges. Interactions v. 15 n.1, 59-60.

[22] Marsden, G., Maunder, A., Parker, M. (2008). People are People, but Technology is not Technology. Phil. Trans. R. Soc. A. 366, 3795-3804. 
[23] Munteanu, C. et al. (2010) ALEX: Mobile Language Assistant for Low-Literacy Adults, Proc. MobileHCI, pp. 427-430.

[24] Munteanu, C. et al. (2011). Showing off your mobile device: Adult literacy learning in the classroom and beyond. Proc MobileHCI, pp. 95-104.

[25] Munteanu, C. et al. (2012). A Tale of Two Studies: Challenges in Field Research with Lowliteracy Adult Learners in a Developed Country. Proc. ACM SIGCHI (CHI'12), pp. 489-504.

[26] Ogata, H. and Yano, Y. (2004). Knowledge awareness map for computer-supported ubiquitous language-learning. Proc. 2nd Int. Workshop on Wireless and Mobile Technologies in Education, pp. 19-26.

[27] Oreglia, E., Liu, Y., Zhao, W. (2011). Designing for Emerging Rural Users: Experiences from China. HCI 2011 ACM Press, pp. 1433-1436.

[28] Paredes, R.G.J., et al. (2005) LOCH: supporting informal language learning outside the classroom with handhelds. Proc. 3rd Int. Workshop on Wireless and Mobile Technologies in Education, pp. 182-186

[29] Petersen, S.A. \& Markiewicz, J.K. (2008) PALLAS: Personalised Language Learning on Mobile Devices. Proc 5th Int. Conf. on Wireless, Mobile, and Ubiquitous Technology in Education, pp. 52-59.

[30] Ramachandran, D., Das, P.D., Canny, J., Cutrell, E. (2010). Mobile-izing Health Workers in Rural India. Proc. of ACM SIGCHI (CHI'10), pp. 1889-1898.

[31] Satchell, C. and Dourish, P. (2009). Beyond the User: Use and Non-Use in HCI. Proc. OzCHI 2009, 9-16.

[32] Siegel, D.A. and Dray, S.M. (2006) Analyzing Qualitative Data from Field Studies. CHI Course Notes.

[33] Stats Canada. (2003) Building on our Competencies: Canadian Results of the International Adult Literacy and Skills Survey. Human Resources and Skills Development Canada Catalogue, no. 89-617-XIE

[34] Stewart, G., Kerr, A. (2010). A Backgrounder on Apprenticeship Training in Canada. Canadian Apprenticeship Journal, Vol. 1, 1-18.

[35] Thornton, P. and Houser, C. (2005) Using mobile phones in English education in Japan. J. of Computer Assisted Learning, vol. 21, no. 3, pp. 217-228

[36] Todd, R.W. and Tepsuriwong, S. (2008) Mobile Mazes: Investigating a Mobile Phone Game for Language Learning. CALL - EJ Online, vol. 10 no. 1

[37] UNESCO. (2002) Trouble with Reading. UNESCO Education Report

[38] Watanabe, W.M. (2009) Facilita: Reading Assistance for Low-Literacy Readers. Proc. SIGDOC, pp. 29-36

[39] Watanabe, W.M. et al. (2010) Adapting web content for low-literacy readers by using lexical elaboration and named entities labeling. Proc. W4A, pp. 8:1-8:9. 